\title{
Redundancy of myostatin and growth/differentiation factor I I function
}

\author{
Alexandra C McPherron*1, Thanh V Huynh² and Se-Jin Lee*2
}

\author{
Address: ${ }^{1}$ Genetics of Development and Disease Branch, National Institute of Diabetes and Digestive and Kidney Diseases, National Institutes of \\ Health, 9000 Rockville Pike, Bethesda, MD 20892, USA and 2Department of Molecular Biology and Genetics, Johns Hopkins University School \\ of Medicine, 725 North Wolfe Street, Baltimore, MD 21205, USA \\ Email: Alexandra C McPherron* - mcpherrona@niddk.nih.gov; Thanh V Huynh - thuynh@jhmi.edu; Se-Jin Lee* - sjlee@jhmi.edu \\ * Corresponding authors
}

Published: 19 March 2009

BMC Developmental Biology 2009, 9:24 doi:10.1186/I47|-213X-9-24

This article is available from: http://www.biomedcentral.com/I47I-2/3X/9/24

(C) 2009 McPherron et al; licensee BioMed Central Ltd.

This is an Open Access article distributed under the terms of the Creative Commons Attribution License (http://creativecommons.org/licenses/by/2.0), which permits unrestricted use, distribution, and reproduction in any medium, provided the original work is properly cited.
Received: 3 November 2008

Accepted: 19 March 2009

\begin{abstract}
Background: Myostatin (Mstn) and growth/differentiation factor II (GdfII) are highly related transforming growth factor $\beta$ (TGF $\beta$ ) family members that play important roles in regulating embryonic development and adult tissue homeostasis. Despite their high degree of sequence identity, targeted mutations in these genes result in non-overlapping phenotypes affecting distinct biological processes. Loss of Mstn in mice causes a doubling of skeletal muscle mass while loss of GdfI I in mice causes dramatic anterior homeotic transformations of the axial skeleton, kidney agenesis, and an increase in progenitor cell number in several tissues. In order to investigate the possible functional redundancy of myostatin and $\mathrm{Gdfl}$ I, we analyzed the effect of eliminating the functions of both of these signaling molecules.
\end{abstract}

Results: We show that Mstn ${ }^{-/-} \mathrm{GdfI} \mathrm{I}^{-/-}$mice have more extensive homeotic transformations of the axial skeleton than $\mathrm{Gdfl} /$-/- mice in addition to skeletal defects not seen in single mutants such as extra forelimbs. We also show that deletion of GdfI I specifically in skeletal muscle in either Mstn ${ }^{+/}$ + or Mstn-/- mice does not affect muscle size, fiber number, or fiber type.

Conclusion: These results provide evidence that myostatin and $\mathrm{GdfI} I$ have redundant functions in regulating skeletal patterning in mice but most likely not in regulating muscle size.

\section{Background}

Myostatin (Mstn) and growth/differentiation factor 11 (Gdf11) are highly related members of the transforming growth factor $\beta$ (TGF $\beta$ ) superfamily of secreted growth and differentiation factors. Like other TGF $\beta$ family members, myostatin and Gdf11 precursor proteins are proteolytically processed to form biologically-active carboxyterminal dimers. Myostatin and Gdf11 share 90\% amino acid identity in this carboxy-terminal region which places them in their own TGF $\beta$ family subgroup. They also have similar signaling pathways; both bind the activin type IIB receptor (Acvr2b, also known as ActRIIB) and activate the intracellular mediator Smad 2/3 pathway [1-5], and both are antagonized by follistatin, a secreted glycoprotein that can bind several TGF $\beta$ family members $[2,6-9]$.

Mstn is predominantly expressed in developing and adult skeletal muscle [10]. We previously reported the disruption of the Mstn gene by gene targeting in mice and showed that myostatin normally functions as a negative regulator of skeletal muscle mass [10]. Most Mstn-/- muscles are approximately double the mass of $\mathrm{Mstn}^{+/+}$muscles 
due to both hyperplasia and hypertrophy of myofibers [10]. Subsequently, naturally occurring Mstn gene mutations were found in cattle, dogs, sheep, and one child with increased muscle mass indicating conservation of myostatin function in mammals [11]. Injection of myostatin antagonists also causes a significant increase in muscle mass in normal and dystrophic adult mice demonstrating that myostatin function is not restricted to developmental stages [12-17]. These results suggest that inhibition of myostatin may be a promising therapeutic target for treating muscle wasting diseases.

We also reported the deletion of the Gdf11 gene by gene targeting in mice [18]. In contrast to Mstn $\%$ mice, Gdf $11 \%$ mice display anterior homeotic transformations of the axial skeleton wherein the identity of posterior vertebrae are transformed into those of more anterior vertebrae [18]. Anterior/posterior (A/P) axial mesoderm identity is thought to be established by differential combinatorial expression of Hox genes that are induced as cells move through the primitive streak during gastrulation [19]. Consistent with this model, Gdf11 is strongly expressed in the primitive streak and tail bud $[7,18,20]$, and Gdf11\% mice have altered Hox gene expression along the $\mathrm{A} / \mathrm{P}$ axis [18]. In addition to the axial skeleton, defects in Gdf11\% mice are found in other tissues where Gdf11 is expressed. For instance, Gdf11\%-mice have renal agenesis, an increase in the number of islet progenitors in the pancreas, an increase in the number of neurons and neuronal progenitors in the olfactory epithelium, and an increase in the number of retinal ganglion cells and reduction in the number of photoreceptors and amacrine cells in the retina [21-25].

Although the phenotypes caused by each gene disruption appear to be non-overlapping, the high sequence identity and similarity in signaling mechanisms suggest the two factors may be functionally redundant. In addition, Mstn is expressed transiently in the primitive streak in the chick [26]. We therefore decided to test whether myostatin has a role in determining $\mathrm{A} / \mathrm{P}$ positional identity of the axial skeleton. In a similar vein, Gdf11 has been shown to inhibit myogenesis in chick limb mensenchyme cultures [27], so we also sought to test whether Gdf11 has a role in regulating skeletal muscle mass. Our data show that myostatin and Gdf11 have redundant functions in regulating skeletal patterning in mice but most likely not in regulating muscle size.

\section{Results and discussion}

Skeletal patterning in Mstn-l- GdfI I-l- mice

We crossed double heterozygous mice $\left(\mathrm{Mstn}^{+/-} \mathrm{Gdf} 11^{+/-}\right)$ to produce Mstn $\%$ Gdf11\% mice to examine redundancy of myostatin and Gdf11 function during development. Mstn/- Gdf11\% mice were born at the expected ratio (data not shown), but none were found alive. While most Gdf11\% mutants have renal agenesis and cleft palate [22], these phenotypes were fully penetrant in Mstn $\%$ Gdf11\% mice. To analyze skeletal alterations in double mutants, we performed skeleton preps on newborn mice to determine vertebral identity. Wild-type mice commonly have 13 thoracic and 6 lumbar vertebrae while Gdf11\% mice typically have 18 thoracic and 8 lumbar vertebrae [18]. Gdf11\% offspring from crosses of double heterozygotes had 17-19 thoracic and 7-9 lumbar vertebrae as expected (Tables 1 and 2, Figure $1 \mathrm{~A}$ ). In contrast, skeletons of $\mathrm{Mstn}-/$ - mice had a similar pattern as the majority of wild-type mice with 13 thoracic and 6 lumbar vertebrae (Tables 1 and 2). Deletion of one allele of Mstn in Gdf11\% mice (Mstn ${ }^{+/}$Gdff1\%) also had no effect on thoracic or lumbar vertebral number in comparison with Gdf11\% mice (Tables 1 and 2). Loss of both alleles of Mstn in Gdf11\% mice, however, resulted in a much more severe phenotype. Most Mstn $\%$ Gdf11\% mice had 20 rather than 18 thoracic vertebrae although in 4 out

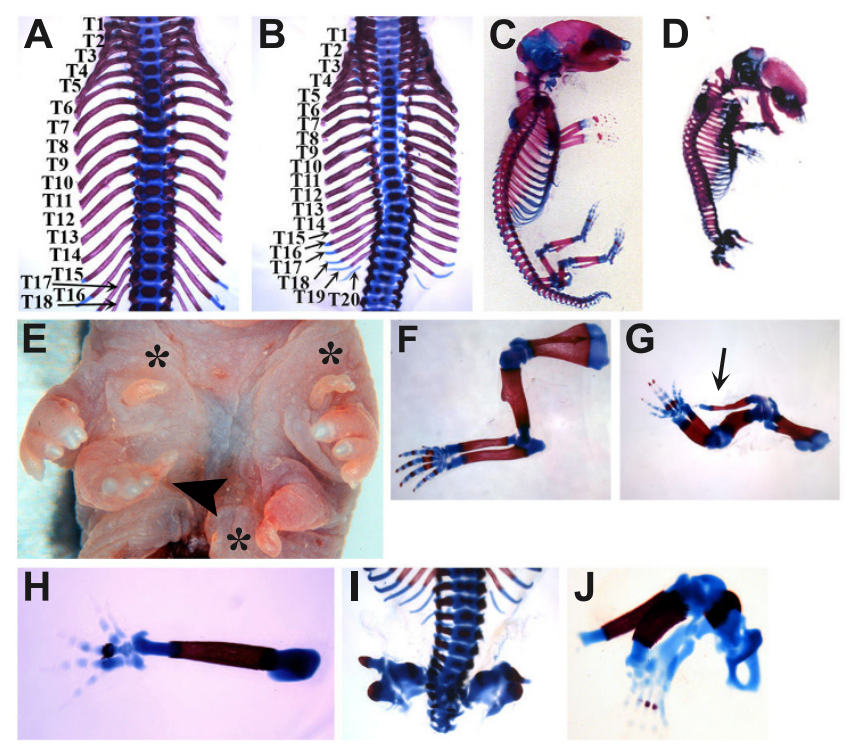

Figure I

Skeletal defects in Mstn-/- GdfI I-/- newborn mice. Thoracic region of GdfI I-/- (A) and Mstn ${ }^{-/-}$Gdfl I-/- (B) mutants showing the increase in rib number. Note that the anterior homeotic transformations of thoracic vertebrae are more extensive in the Mstn ${ }^{-/}$GdfI I-/- mutant (20 ribs) versus the single GdfI I-/-mutant ( 8 ribs). Whole skeleton preparation of GdfI I-/- (C) and Mstn ${ }^{-/-}$GdfI I-/- (D) pups. (E) Ventral torso of newborn double mutant showing multiple projections from the skin $(*)$ and an extra limb (arrowhead). Forelimb skeleton preparations of GdfI I-/- (F) and Mstn ${ }^{-/-}$GdfI I-/- (G and $\mathrm{H}$ ) pups showing an extra bone emanating from the shoulder of the double mutant (G, arrow) and an extra limb $(H)$. The limb in $(G)$ has been rotated for a better view of the extra bone. (I and J) Hindlimb phenotypes of Mstn ${ }^{-/-}$Gdf I I-/pups. Note the truncation of the vertebral column (I) and malformation of the ilieum and all leg bones $(J)$. 
Table I: Thoracic vertebral number from newborn mice

\begin{tabular}{|c|c|c|c|c|c|c|c|c|c|}
\hline \multirow{3}{*}{$\begin{array}{l}\text { Mstn } \\
\text { GdfI I }\end{array}$} & \multicolumn{9}{|c|}{ Genotype } \\
\hline & $+/+$ & $+/+$ & $+/+$ & $+/-$ & $+/-$ & $+/-$ & $-/-$ & $-/-$ & $-/-$ \\
\hline & $+/+$ & $+/-$ & $-/-$ & $+/+$ & $+/-$ & $-/-$ & $+/+$ & $+/-$ & $-/-$ \\
\hline Total number of thoracic vertebrae & \multicolumn{9}{|c|}{ Number of animals } \\
\hline 13 & 10 & - & - & 10 & - & - & 10 & I & - \\
\hline 14 & - & 10 & - & - & 10 & - & - & 9 & - \\
\hline 15 & - & - & - & - & - & - & - & - & - \\
\hline 16 & - & - & - & - & - & - & - & - & - \\
\hline 17 & - & - & - & - & - & - & - & - & - \\
\hline $17+18$ & - & - & I & - & - & - & - & - & - \\
\hline 18 & - & - & 8 & - & - & 7 & - & - & - \\
\hline $18+19$ & - & - & - & - & - & I & - & - & - \\
\hline 19 & - & - & I & - & - & 2 & - & - & 1 \\
\hline $19+20$ & - & - & - & - & - & - & - & - & I \\
\hline 20 & - & - & - & - & - & - & - & - & 11 \\
\hline $20+21$ & - & - & - & - & - & - & - & - & 4 \\
\hline
\end{tabular}

Numbers of mice displaying a given number of vertebrae are shown. Some mice have left-right asymmetry of the most posterior thoracic vertebra with a rib on one side and a lumbar phenotype on the other. These are designated by a + sign between 2 numbers indicating the number of ribs for each side.

Table 2: Lumbar vertebral number from newborn mice

\begin{tabular}{|c|c|c|c|c|c|c|c|c|c|}
\hline \multirow{3}{*}{$\begin{array}{l}\text { Mstn } \\
\text { GdfII }\end{array}$} & \multicolumn{9}{|c|}{ Genotype } \\
\hline & $+/+$ & $+/+$ & $+/+$ & $+/-$ & $+/-$ & $+/-$ & $-/-$ & $-/-$ & $-/-$ \\
\hline & $+/+$ & $+/-$ & $-/-$ & $+/+$ & $+/-$ & $-/-$ & $+/+$ & $+/-$ & $-/-$ \\
\hline Total number of lumbar vertebrae & \multicolumn{9}{|c|}{ Number of animals } \\
\hline 5 & 3 & - & - & 3 & - & - & - & - & \\
\hline $5+6$ & 1 & - & - & - & - & - & - & - & \\
\hline 6 & 6 & 10 & - & 7 & 10 & - & 10 & 9 & \\
\hline $6+7$ & - & - & - & - & - & - & - & - & \\
\hline 7 & - & - & 1 & - & - & I & - & 1 & \\
\hline $7+8$ & - & - & 1 & - & - & - & - & - & \\
\hline 8 & - & - & 5 & - & - & 5 & - & - & \\
\hline $8+9$ & - & - & 2 & - & - & - & - & - & \\
\hline 9 & - & - & 1 & - & - & 3 & - & - & \\
\hline $9+10$ & - & - & - & - & - & 1 & - & - & \\
\hline
\end{tabular}

Numbers of mice displaying a given number of vertebrae are shown. Some mice have left-right asymmetry of the most posterior lumbar vertebra with a lumbar phenotype on one side and a sacral phenotype the other. These are designated by a + sign between 2 numbers indicating the number of vertebrae possessing a lumbar appearance for each side. Asymmetric vertebrae with a thoracic and lumbar phenotype were counted as thoracic (Table I). Number of lumbar vertebrae could not be determined for Mstn-l- GdfI I-l- mice.

of 17 double mutant pups the transformations were even more dramatic (Figure 1B, Table 1). In these 4 mice, the most posterior thoracic vertebra was asymmetric with a $21^{\text {st }}$ rib on one side and a lumbar-like phenotype on the other (Table 1 and 2). The extent of the anterior homeotic transformations in the thoracic region of Mstn $\%$ Gdf11\% mice were greater than any we have seen in $G d f 11 \%$ mice.

The skeletal regions posterior to the thoracic region were also altered in mutant mice. As previously described [18], the sacral vertebrae were present in Gdf11-/- mice, but the axis was truncated in the caudal region resulting in an overall decrease in the number of vertebral segments and the loss of most of the tail with only a few deformed vertebrae remaining (Figure 1C). In Mstn-/- Gdf11-/- mice, there were even fewer post-thoracic vertebrae with an average of 10 post-thoracic segments, the most posterior of which were malformed (Figure 1D and 1I). The sacral vertebrae were not identifiable in Mstn-/- Gdf11-/- mice making it difficult to tell which vertebra was the most pos- 
terior lumbar vertebra (Figure 1I). The increase in severity of the Gdf11 null phenotype throughout the vertebral axis in the absence of Mstn demonstrates that myostatin and Gdf11 play redundant roles in patterning and development of the axial skeleton.

Mstn $\%$ Gdf11\% pups also had other skeletal defects not seen in $G d f 11 \%$ mutants. The frontal bones curved anteriorly to meet the nasal bone which gave the skull vault a rounded shape compared to Gdf11\% mice (Figure 1C and $1 \mathrm{D}$ and Additional file $1 \mathrm{~A}$ and $1 \mathrm{~B}$ : Cranial and forelimb digit skeletal defects in Mstn $\%$ Gdf11\% newborn mice). Although Gdf11 is expressed in the developing limb buds $[7,20]$, no defects were detected in limbs of Gdf11\% mutants (Figure 1C and 1F). Mstn $\%$ Gdf11\% mice, however, had severe limb defects. Many Mstn $\%$ Gdf11\% mice had small projections emanating from the skin on the ventral surface of the torso, some of which were filled with a single rod of cartilage (Figure 1E). In Mstn $\%$ Gdf11\%mutants, the long bones of the forelimb were shortened relative to those in Gdf11\% mice and, in 9 out of 18 double mutants, an extra bone projected from shoulder (Figure $1 \mathrm{~F}$ and $1 \mathrm{G}$ ). The most surprising result was a third limb that resembled a forelimb composed of a single unidentifiable long bone with attached digits (Figure $1 \mathrm{E}$ and $1 \mathrm{H})$. This phenotype was found in 6 out of 18 double mutants, two of which also had an extra bone on the contralateral shoulder. In all Mstn $/$ Gdf11\%-mutants, the forelimbs in the normal position displayed digital patterning defects including a sixth digit, which appeared to be similar to digit $\mathrm{V}$, and syndactyly of digits III and IV consistent with the expression of Gdf11 in the interdigital region where programmed cell death would normally occur $[7,20]$ (Figure 1G and Additional file 1C and 1D: Cranial and forelimb digit skeletal defects in Mstn \% Gdf11\% newborn mice). No other genotypes displayed any of these limb defects.

The hindlimbs in Mstn $\%$ Gdf11\% mice displayed very different malformations than the forelimbs. The hindlimbs were small and deformed although what appeared to be elements of both proximal and distal structures, pelvic bones, long bones, and digits, were seen in most double mutants (Figure 1D, I and 1J). These results suggest some proximal/distal patterning was maintained despite inhibition of hindlimb bud outgrowth.

This is the first demonstration that myostatin and Gdf11 are both required for limb development and axial skeletal development and patterning. The effects of deletion of both factors on the axial skeleton was greater than for loss of Gdf11 alone most likely due to the transient expression of Mstn in the posterior primitive streak overlapping with Gdf11 expression [26]. We presume that the extra limbs seen in the double mutant mice result from an expansion of the limb field, but clearly additional studies will be required to fully understand the molecular basis for the formation of the extra limb buds.

\section{Skeletal muscle in GdfI I mutants}

We also sought to determine whether myostatin and Gdf11 are functionally redundant with respect to control of muscle mass. The increase in muscle mass in Mstnmice is not present at birth (A.C.M. and S-J.L., unpublished observations) so the neonatal death of $G d f 11^{-}$and Mstn- Gdf11\%-mice precludes a comparison of the skeletal muscle phenotype. We therefore generated a targeting construct containing a conditional deletion allele of the Gdf11 gene by inserting loxP recombination sites into intron 1 and flanking a neo gene downstream of the Gdf11 3' UTR (Gdf11flox-neo) (Figure 2A and 2B). Following homologous recombination in embryonic stem (ES) cells and injection of the targeted cells into blastocysts, we obtained chimeric mice that transmitted the Gdf11 flox-neo allele through the germline. Mice carrying the Gdf1 1 flox-neo allele were then crossed to EIIa-Cre transgenic mice to generate Gdf1 flox/+ mice carrying one upstream and one downstream loxp site after removal of the neo gene. Recombination at the remaining loxP sites would be predicted to delete exons 2 and $3\left(G d f 11^{\Delta 2-3}\right)$, which would remove the biologically-active carboxy-terminal domain. To demonstrate that recombination of loxP sites produced a null allele, we generated germline recombination of lox $\mathrm{P}$ sites in Gdf1 $1^{f l o x /+}$ mice (Gdf1 $1^{\Delta 2-3 /+}$ ). Skeletal analysis performed on offspring from $G d f 11^{\Delta 2-3 /+}$ matings showed that $G d f 11^{\Delta 2-3 /+}$ and $G d f 11^{\Delta 2-3 / \Delta 2-3}$ mice had 1 and 5 extra thoracic vertebrae, respectively (data not shown). These thoracic vertebral numbers are identical to that of Gdf $11^{+/}$ - and Gdf11\% mice [18] confirming that recombination of loxP sites in the Gdf11 flox allele results in a null allele.

For skeletal muscle-specific recombination of loxP sequences, a Cre deletor transgenic line was made using the myosin light chain $1 / 3$ promoter/enhancer (MLC-Cre) (Figure 2A). As expected, Cre expression in this line was restricted to skeletal muscle (Figure 2C). MLC-Cre mice were crossed to $G d f 11^{f l o x /+}$ mice and then backcrossed to Gdf1 $11^{f l o x /+}$ mice or crossed to Gdf11+/- from the original knockout line. Recombination of Gdf1 flox was detected specifically in skeletal muscle genomic DNA (Figure 2D). In Gdf1 flox/- muscle, Cre-mediated recombination resulted in a near complete reduction in skeletal muscle Gdf11 expression (Figure 2E).

Body weight and muscle mass were measured in all 8 possible genotypes produced from crosses of $G d f 11^{f l o x} /+M L C$ Cre and Gdf11+/- mice. There were no differences in body weight or muscle mass between $G d f 11^{+/+}, G d f 11^{+/+} M L C$ Cre, Gdf11+/-, Gdf11+/- MLC-Cre, Gdf1 $11^{f l o x /+}$, or Gdf1 flox/+ MLC-Cre mice demonstrating that there was no hetero- 
A

Genomic locus

Gdf11 flox-neo allele

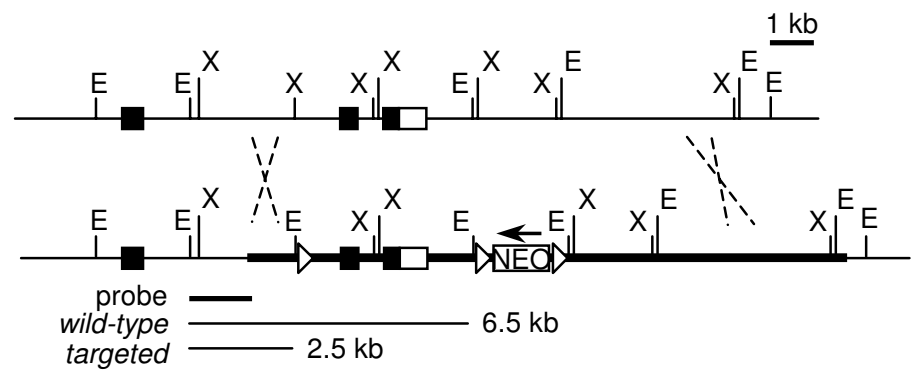

Gdf11 flox allele

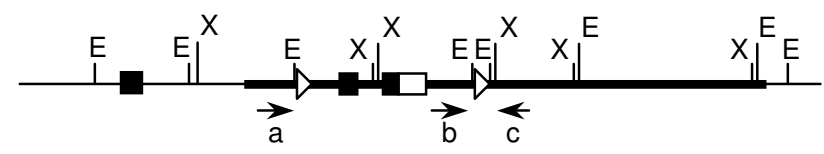

Gdf11 $\Delta 2-3$ allele

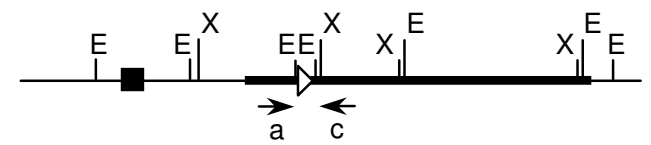

$M L C$-Cre transgene
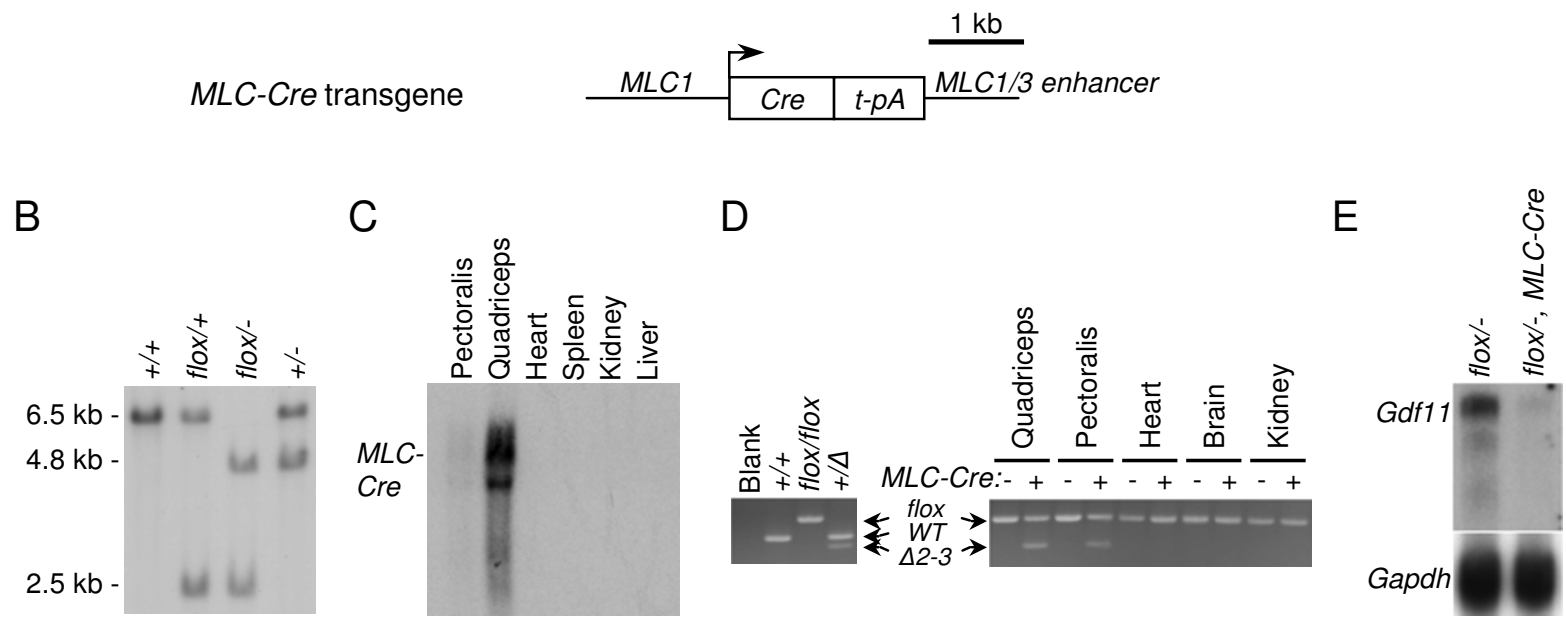

\section{Figure 2}

Muscle-specific targeting of GdfI I gene. (A) Representation of targeting strategy. The 3 exons are shown as boxes with coding sequences shaded black and the 3'UTR open. The targeting construct is represented by a thick line which contains loxP sequences with EcoRI restriction sites inserted into the second Xbal site in exon I and flanking a neo gene inserted into the EcoRI site downstream of the 3'UTR. Cre-mediated recombination of the loxP sites flanking the neo gene results in a GdfI Iflox allele. Recombination of the $\mathrm{Gdfl}$ I flox allele generates the $G d f|| \Delta 2-3$ allele. Oligonucleotide primers used for distinguishing alleles are labeled a, b, and c. A skeletal muscle-specific Cre expressing transgene was constructed using the $\mathrm{MLCl}$ promoter/I/3 enhancer and an SV40 $t$ antigen intron and poly adenylation signal. (B) Southern blot showing detection of GdfI I+, GdfI Iflox, and GdfI I-(null, from the original knockout line) alleles. (C) Northern blot analysis of MLC-Cre transgene expression in pectoralis and quadriceps muscles but not in other tissues. (D) Detection of Gdfl I alleles in genomic DNA in Gdfl Iflox/floxand Gdfl Iflox/ floxMLC-Cre mice by PCR (right panel). Recombination was detected in quadriceps and pectoralis muscles of GdfI I flox/floxMLC-Cre mice but not in heart, brain, or kidney. No recombination was seen in Gdfl I flox/floxmice. Left panel shows control reactions. (E) Northern blot analysis of skeletal muscle GdfI I expression showing a strong decrease in expression in GdfI Ifloxl-MLC-Cre muscle compared to GdfI I floxl-muscle.

zygous or transgene phenotype (data not shown). We next performed an examination of the muscle phenotype of Gdf1 $1^{\text {flox/- }}$ and Gdf1 $1^{\text {flox/- }}$ MLC-Cre mice. There was no statistically significant difference in body weight or muscle mass between Gdf11 flox/- and Gdf11 flox/- MLC-Cre mice (data not shown and Figure 3A).
Skeletal muscles vary in their composition of fiber types which differ in metabolic and contractile properties [28]. In mice, type I fibers are slow contracting and oxidative, type IIB fibers are fast contracting and glycolytic, and type IIA and IID/X fibers are intermediate in phenotype. Mstn/- mice have an overall increase in the number of muscle 

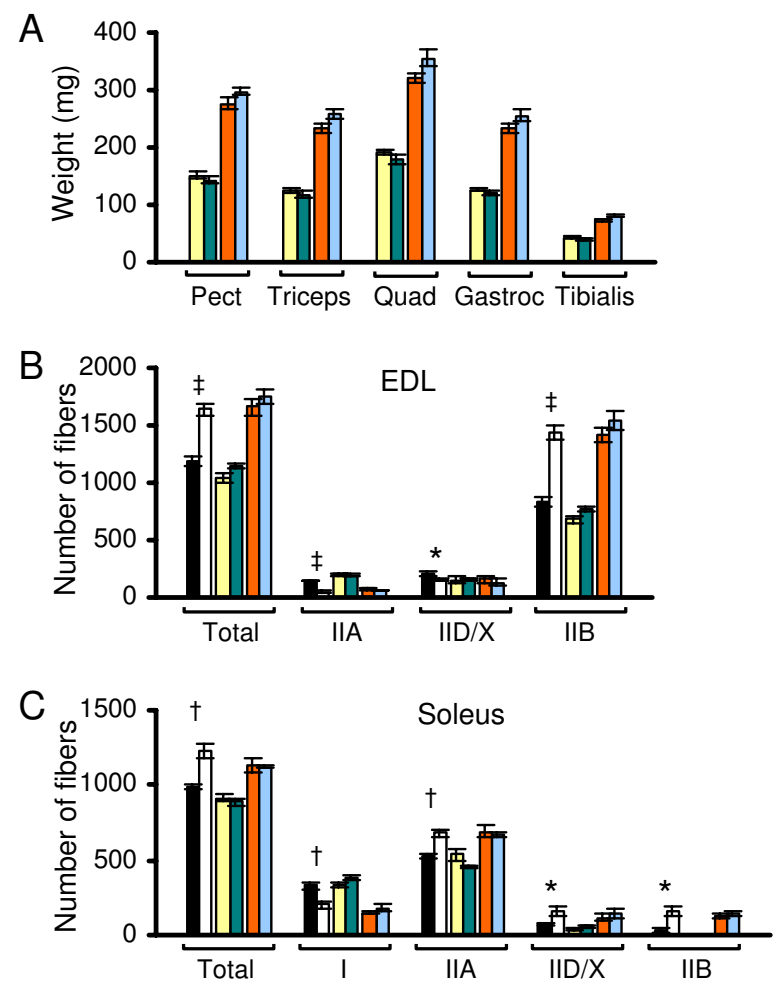

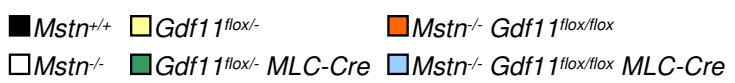

\section{Figure 3}

Muscle weight, fiber number, and fiber type in skeletal muscle-specific GdfI I mutant mice in a Mstn wildtype or null background. (A) Weight of pectoralis, triceps, quadriceps, gastrocnemius/plantaris, and tibialis anterior muscles $(n=7-12)$. (B) Number of total, IIA, IID/X, and IIB fibers in the EDL muscle $(n=3-4)$. EDL muscles had on average less than 4 type I fibers in all genotypes so type I data are not shown. (C) Number of total, type I, IIA, IID/X, and IIB fibers in the soleus muscle $(n=3-5)$. Skeletal muscle-specific GdfI I deletion had no effect on muscle mass, fiber number, or fiber type unlike Mstn deletion ( $* P<0.05$, $† P<$ $0.01, \pm P<0.001$ ). Data are mean \pm s.e.

fibers and alterations in the proportions of the different fiber types compared to $\mathrm{Mstn}^{+/+}$mice resulting in a shift toward more glycolytic muscle $[10,29,30]$. To determine whether Gdf11 regulates fiber number or type, we counted the number of each of the four fiber types in Gdf1 flox/- and Gdf11 flox/- MLC-Cre mice from the widest part of each muscle. We chose a fast muscle, the extensor digitorum longus (EDL), and a slow muscle, the soleus, for analysis because they have been previously shown to have alterations in fiber number and type in Mstn-/- mice $[29,30]$. Unlike muscle in Mstn ${ }^{-/}$mice, there was no difference in the total number of fibers in either the EDL or the soleus muscles between Gdf11 flox/- and Gdf11 flox/-MLC-Cre mice (Figure 3B and 3C). Fiber type composition in Gdf1 $1^{\text {flox/- }}$ and Gdf1 $1^{\text {flox/- }}$ MLC-Cre mice was not significantly different either (Figure 3B and 3C).

Because a role for myostatin in axial skeletal patterning was found only in the absence of Gdf11, we asked whether a phenotype caused by loss of Gdf11 in muscle might be present in the absence of myostatin. Mstn-/Gdf1 $1^{\text {flox/flox }}$ mice were crossed to Mstn-/- Gdf11 flox/flox MLCCre mice, and body weight and muscle mass were measured in offspring at 8 weeks and 6 months of age. There were no significant differences in body weight or muscle mass found between genotypes at either age (data not shown and Figure 3A). Next, we determined fiber number and fiber composition. There were no significant differences in the total number of fibers or in the number of individual fiber types in EDL or soleus between Mstn-/Gdf1 $1^{\text {flox/flox }}$ and Mstn $/-$ Gdf1 $1^{\text {flox/flox }}$ MLC-Cre mice (Figure $3 \mathrm{~B}$ and $3 \mathrm{C})$.

Our results provide evidence that that myostatin and Gdf11 have redundant functions in regulating skeletal development and patterning but not skeletal muscle size. We certainly cannot rule out the possibility that sufficient Gdf11 function is maintained by the small amount of Gdf11 expression remaining in the mutant muscles or that Gdf11 may have a function in developing skeletal muscle at early stages of development prior to activation of the $M L C$ promoter. Gdf11 is detectable in serum [31] so we also cannot rule out the possibility that Gdf11 produced by other tissues not affected by muscle-specific recombination contributes to muscle mass regulation.

Nevertheless, the lack of an effect of these genetic manipulations of Gdf11 signaling on muscle mass was somewhat unexpected because myostatin and Gdf11 are highly homologous and because the function of myostatin in regulating skeletal muscle mass is clearly redundant with that of other TGF $\beta$ family members. Injection of a soluble Acvr2b receptor causes a $20 \%$ increase in muscle mass in $\mathrm{Mstn}{ }^{-/}$mice demonstrating that at least one other ligand in addition to myostatin is a negative regulator of skeletal muscle size [15]. This other Acvr2b-binding ligand(s) must also be inhibited by follistatin; Mstn $/$ mice have double the muscle mass of $\mathrm{Mstn}^{+/+}$mice while Mstn /- mice overexpressing a muscle-specific follistatin transgene have quadruple the skeletal muscle mass of $\mathrm{Mstn}^{+/+}$mice [32]. Several other TGF $\beta$ superfamily members are potential candidates for myostatin functional redundancy. In addition to Gdf11, other family members, such as the activins and some bone morphogenetic proteins, bind Acvr2b and follistatin [31,33-36]. Myostatin, Gdf11, activin, and some BMPs can be isolated from serum using Acvr2b affinity purification [31]. The affinity of the BMPs for fol- 
listatin, however, is considerably lower than that of activin, myostatin, and Gdf11 $[8,9]$. Additionally, the activins inhibit differentiation of $\mathrm{C} 2 \mathrm{C} 12$ myoblasts and skeletal muscle precursors in the chick limb and would therefore be likely candidates for functional redundancy with myostatin $[31,37]$. It is also possible there are multiple TGF $\beta$ family members that are negative regulators of muscle mass in addition to myostatin that individually have only small effects on muscle growth. If so, deletion or inhibition of these factors might cause a measurable increase in muscle mass only in the absence of myostatin and other redundant ligands.

Not only are the ligands redundant, myostatin and Gdf11 receptor function is also redundant. Although the affinity of myostatin for activin type II receptor (Acvr2, also known as ActRIIA) is low [2,5], Acvr2\% mice, like Acvr $2 b^{-1}$ - mice, have a small increase in skeletal muscle mass relative to Mstn-/- mice [15]. This suggests that both Acvr2 and Acvr2b are receptors for myostatin in vivo. Similarly, Acvr $2 \mathrm{~b} \%$ mice have anterior homeotic transformations of the axial skeleton that are milder than those seen in Gdf11/- mice [38]. The Acvr $2^{+/-}$Acvr $2 b^{-/}$axial skeletal pattern closely phenocopies that of Gdf11\% mice [3] suggesting that both Acvr2 and Acvr2b are receptors for Gdf11. These data suggest therapeutic strategies that target both Acvr2 and Acvr2b receptor function or myostatin and other TGF $\beta$ growth factors with similar functions would lead to greater increases in muscle mass than targeting either Acvr2b or myostatin alone.

\section{Conclusion}

Our results demonstrate that myostatin and Gdf11 have redundant functions in regulating skeletal patterning in mice. We did not, however, find evidence that Gdf11 is the TGF $\beta$ family member that is redundant to myostatin in regulation of skeletal muscle size. To determine the therapeutic strategy that results in the greatest potential increase in muscle mass, it will be important to identify the other TGF $\beta$ family members that negatively regulate skeletal muscle mass.

\section{Methods \\ RNA isolation, Northern analysis}

Total RNA was isolated from 8 week old animals from a pool of pectoralis, quadriceps, triceps, gastrocnemius, plantaris, and tibialis anterior muscles using Trizol reagent (Invitrogen). PolyA+ RNA was isolated from total RNA using an Oligotex mRNA kit (Qiagen). For Northern blot analysis, $5 \mu \mathrm{g}$ mRNA was electrophoresed, blotted to GeneScreen Plus (Perkin Elmer), and hybridized according to the manufacturer's instructions using a probe corresponding to the carboxy-terminal region of Gdf11 in exon 3. After the signal decayed, the same blot was probed with Gapdh as a loading control.

\section{Muscle-specific targeting of GdfI I}

All animal experiments were approved by the Institutional Animal Care and Use Committees of the Johns Hopkins University School of Medicine or NIDDK. To make muscle-specific Cre expressing mice, the coding sequence of Cre recombinase modified to contain Kozak consensus sites was inserted into the MDAF2 vector containing the myosin light chain promoter and $1 / 3$ enhancer and SV40 processing sites [39]. To make the Gdf11 conditional allele, $3 \operatorname{lox} \mathrm{P}$ sites were inserted in intron 1 and flanking a neo gene downstream of the 3' UTR (Figure 2A) and used to replace the endogenous locus by gene targeting in ES cells. Microinjections and blastocyst injections were carried out by the Johns Hopkins School of Medicine Transgenic Core Facility. Offspring of male Gdf11 flox-neo/+ chimeras mated to C57BL/6 females were crossed to EIIa-Cre transgenic mice in the FVB background (The Jackson Laboratory) to generate males mosaic for loxP recombinations [40]. These males were mated to C57BL/ 6 females and screened by PCR for loss of the neo gene and retention of Gdf11 exons 2 and 3 (Gdf11flox/+). Gdf11flox/++ mice were crossed once more to C57BL/ 6 mice, and EIIA-Cre negative Gdf1 $11^{f l o x /+}$ mice were crossed to MLC-Cre mice to generate Gdf1 $11^{\text {flox } /+}$ MLC-Cre mice. Gdf1 $11^{f l o x} /+$ MLC-Cre mice were mated to $G d f 11^{+/-}$in a $129 / \mathrm{Sv}$ genetic background from the original knockout line and to Mstn+/- in the C57BL/6 genetic background. Gdf1 $11^{f l o x} /+M s t n^{+/-} M L C$-Cre mice were crossed to Gdf1 flox/

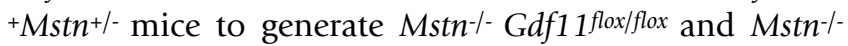
Gdf11 flox/flox MLC-Cre mice which were interbred to produce animals for analysis. To show that $G d f 11^{\Delta 2-3}$ is a null allele, Gdf11 $11^{\Delta 2-3 /+}$ mice with germline recombination of the Gdf $11^{f l o x}$ allele were obtained using Cre/Esr1 mice [41] purchased from The Jackson Laboratory.

\section{Genotyping}

ES cell targeting and F1 offspring were analyzed by Southern blot after EcoRI digestion of genomic DNAs using the same probe as in ref. 18. EcoRI fragment sizes were $6.5 \mathrm{~kb}$ for wild-type and $2.5 \mathrm{~kb}$ targeted Gdf11 flox-neo alleles. Loss of the neo cassette after crosses to EIIA-Cre mice was determined by PCR using primers b and c. Gdf1 $11^{f l o x}, G d f 11^{\Delta 2-3}$, and $\mathrm{Gdf} 11^{+}$alleles were detected by PCR using one reaction containing primers $\mathrm{a}, \mathrm{b}$, and $\mathrm{c}$. Cre lines and Mstn alleles in crosses between $\mathrm{Mstn}{ }^{+/-}$and $\mathrm{Gdf} 11^{f l o x /+}$ mice were genotyped by PCR. All PCR was carried out at an annealing temperature of $55^{\circ} \mathrm{C}$ in buffer containing $10 \mathrm{mM}$ Tris, pH 8.8, $25 \mathrm{mM} \mathrm{KCl}, 1.5 \mathrm{mM} \mathrm{MgCl} 2,0.2 \mathrm{mM}$ of each $\mathrm{dNTP}$, and $0.8 \mu \mathrm{M}$ of each oligonucleotide primer. Oligonucleotide primers used and product sizes: Cre, 505 bp, 5' CCGAAATTGCCAGGATCAGGG $3^{\prime}$ (forward) and $5^{\prime}$ TCGCCATCTTCCAGCAGGCGC 3' (reverse); sstn $^{+}, 220$ bp, 5'-AGAAGTCAAGGTGACAGACACAC-3' (forward) and 5'-GGTGCACAAGATGAGTATGCGG-3' (reverse); Mstn null (in PGK neo cassette), 332 bp, 5'-GGATCG- 
GCCATTGAACAAGATG-3' (forward) and 5'-GAGCAAGGTGAGATGACAGGAG-3' (reverse); Gdf11 alleles,

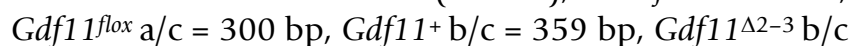
= $393 \mathrm{bp}$, (a) 5'ATGCAGATGGTAATACTTGGG3', (b) 5'AAGGCTTGGGAAGCAGGCAAG-3', and (c) 5'-AGGTATGGTTAGGGTGTGGAG-3'. Gdf11 alleles were genotyped by Southern blot for crosses between the Gdf11 null allele from the original knockout line and Gdf1 $1^{f l o x /+}$ MLC-Cre.

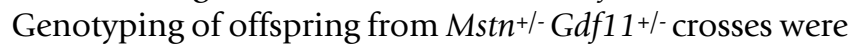
performed by Southern blot as described $[10,18]$.

\section{Analysis of muscles and skeletons}

Soleus and EDL muscles from 8 week old (Gdf1 fllox/-, Gdf11 flox/- MLC-Cre, Gdf1 flox/flox, and Gdf1 flox/flox MLC$\mathrm{Cre})$ or 13 week old $\left(\mathrm{Mstn}^{+/+}\right.$and $\left.\mathrm{Mstn}^{-/}\right)$mice were frozen and $12 \mu \mathrm{m}$ cryostat sections were taken as described [10]. Fibers were counted from the widest part of the muscle belly from $\mathrm{H} / \mathrm{E}$ (soleus) or trichrome (EDL) stained sections. Fiber typing was done on adjacent sections. Primary antibodies were used as dilutions of supernatants from hybridoma cell lines (American Type Culture Collection) and detected with the M.O.M. Peroxidase Kit (Vector Laboratories). Cell lines, dilutions, and fiber types detected were as follows: BA-D5, 1:30, type I; SC-71, 1:5, type IIA; BF-F3， 1:1, type IIB; N2.261， 1:5 plus BF-F3， 1:1, unstained type IID/X. Skeletons of newborn mice were stained as described [18].

\section{Statistical analysis}

Body weight and muscle weights for the eight different genotypes produced from crosses between Gdf11flox/+ MLC-Cre and Gdf11+/- mice were analyzed by single-factor ANOVA with genotype as the factor and were considered significant if $P<0.05$. All other comparisons between two genotypes were analyzed by Student's $t$-test.

\section{Abbreviations}

Acvr2: activin type II receptor; Acvr2b: activin type IIB receptor; Gdf11: growth/differentiation factor 11; MLC: myosin light chain; Mstn: myostatin; TGF $\beta$ : transforming growth factor $\beta$.

\section{Authors' contributions}

S-JL designed and produced the Gdf11 targeting construct. TVH constructed the MLC-Cre transgene and characterized the transgenic lines. ACM carried out ES cell targeting, performed all other experiments, and wrote the manuscript. All authors read and approved the final manuscript.

\section{Authors' information}

Under a licensing agreement between MetaMorphix, Inc. (MMI) and the Johns Hopkins University, the authors are entitled to a share of royalty received by the University on sales of the factors described in this paper. We and the
University own MMI stock, which is subject to certain restrictions under University policy. S-JL, who is the scientific founder of MMI, is a paid consultant to MMI and to Merck on research areas related to the study described in this paper. The terms of these arrangements are being managed by the University in accordance with its conflict of interest policies.

\section{Additional material}

\section{Additional file 1}

Cranial and forelimb digit skeletal defects in $\mathrm{Mstn}^{-/} \mathrm{Gdf11} \%$ newborn mice. (A and B) Skull phenotype of $\mathrm{Gdf} 11 \%$ (A) and $\mathrm{Mstn} \%$ Gdf11\% (B) pups. Double mutants have a rounded frontal bone (arrow). Forelimb digit phenotype of $\mathrm{Gdf} 11 \%$ (C) and $\mathrm{Mstn}^{-} \% \mathrm{Gdf1} 1 \%$ (D) pups. Digit identity is labeled with roman numerals. Note the fusion of digits III and IV and the supernumerary digit $V\left(V^{*}\right)$ in the double mutant. Click here for file

[http://www.biomedcentral.com/content/supplementary/1471213X-9-24-S1.pdf]

\section{Acknowledgements}

We thank N. Brockoff and J. Portas for assistance in maintenance of mouse lines, B. Sauer for the Cre vector, S. Pearson-White for the MDAF2 vector, and the Johns Hopkins Transgenic Core Facility for carrying out the pronuclear and blastocyst injections. This work was supported by grants from the National Institutes of Health (ROIHD35887 and U54AR052646, to S-J.L.) and the Intramural Research Program of the National Institute of Diabetes and Digestive and Kidney Diseases, National Institutes of Health, USA (A.C.M.).

\section{References}

I. Langley B, Thomas M, Bishop A, Sharma M, Gilmour S, Kambadur R: Myostatin inhibits myoblast differentiation by down-regulating MyoD expression. J Biol Chem 2002, 277(5 I):4983 I-49840.

2. Lee S-J, McPherron AC: Regulation of myostatin activity and muscle growth. Proc Natl Acad Sci USA 200I, 98:9306-93II.

3. Oh SP, Yeo CY, Lee Y, Schrewe H, Whitman M, Li E: Activin type IIA and IIB receptors mediate GdfI I signaling in axial vertebral patterning. Genes Dev 2002, 16(21):2749-2754.

4. Philip B, Lu Z, Gao Y: Regulation of GDF-8 signaling by the p38 MAPK. Cell Signal 2005, I 7(3):365-375.

5. Rebbapragada A, Benchabane H, Wrana JL, Celeste AJ, Attisano L: Myostatin signals through a transforming growth factor $\beta$ like signaling pathway to block adipogenesis. Mol Cell Biol 2003, 23(20):7230-7242.

6. Amthor H, Nicholas G, McKinnell I, Kemp CF, Sharma M, Kambadur R, Patel K: Follistatin complexes Myostatin and antagonises Myostatin-mediated inhibition of myogenesis. Dev Biol 2004, 270(I): 19-30.

7. Gamer LW, Wolfman NM, Celeste AJ, Hattersley G, Hewick R, Rosen V: A novel BMP expressed in developing mouse limb, spinal cord, and tail bud is a potent mesoderm inducer in Xenopus embryos. Dev Biol 1999, 208(I):222-232.

8. Schneyer AL, Sidis Y, Gulati A, Sun JL, Keutmann H, Krasney PA: Differential antagonism of activin, myostatin and growth and differentiation factor $I I$ by wild-type and mutant follistatin. Endocrinology 2008, I49(9):4589-4595.

9. Sidis $Y$, Mukherjee A, Keutmann H, Delbaere A, Sadatsuki M, Schneyer A: Biological activity of follistatin isoforms and follistatin-like-3 is dependent on differential cell surface binding and specificity for activin, myostatin, and bone morphogenetic proteins. Endocrinology 2006, I 47(7):3586-3597. 
10. McPherron AC, Lawler AM, Lee SJ: Regulation of skeletal muscle mass in mice by a new TGF- $\beta$ superfamily member. Nature 1997, 387(6628):83-90.

II. Lee SJ: Sprinting without myostatin: a genetic determinant of athletic prowess. Trends Genet 2007, 23(I 0):475-477.

12. Bogdanovich S, Krag TO, Barton ER, Morris LD, Whittemore LA Ahima RS, Khurana TS: Functional improvement of dystrophic muscle by myostatin blockade. Nature 2002, 420(69|4):4|8-42I.

13. Bogdanovich S, McNally EM, Khurana TS: Myostatin blockade improves function but not histopathology in a murine mode of limb-girdle muscular dystrophy 2C. Muscle Nerve 2008, 37(3):308-3। 6.

14. Bogdanovich S, Perkins KJ, Krag TO, Whittemore LA, Khurana TS: Myostatin propeptide-mediated amelioration of dystrophic pathophysiology. FASEB J 2005, I 9(6):543-549.

15. Lee SJ, Reed LA, Davies MV, Girgenrath S, Goad ME, Tomkinson KN, Wright JF, Barker C, Ehrmantraut G, Holmstrom J, et al.: Regulation of muscle growth by multiple ligands signaling through activin type II receptors. Proc Natl Acad Sci USA 2005 102(50): $|8| \mid 7-18122$.

16. Whittemore LA, Song K, Li X, Aghajanian J, Davies M, Girgenrath S, Hill J], Jalenak M, Kelley P, Knight A, et al.: Inhibition of myostatin in adult mice increases skeletal muscle mass and strength. Biochem Biophys Res Commun 2003, 300(4):965-97I.

17. Wolfman NM, McPherron AC, Pappano WN, Davies MV, Song K Tomkinson KN, Wright JF, Zhao L, Sebald SM, Greenspan DS, et al.: Activation of latent myostatin by the BMP-I/tolloid family of metalloproteinases. Proc Natl Acad Sci USA 2003 , I 00(26): I 5842-I5846.

18. McPherron AC, Lawler AM, Lee SJ: Regulation of anterior/posterior patterning of the axial skeleton by growth/differentiation factor I I. Nat Genet 1999, 22(3):260-264.

19. Wellik DM: Hox patterning of the vertebrate axial skeleton. Dev Dyn 2007, 236(9):2454-2463.

20. Nakashima M, Toyono T, Akamine A, Joyner A: Expression of growth/differentiation factor I I, a new member of the BMPI TGF $\beta$ superfamily during mouse embryogenesis. Mech Dev 1999, 80(2): 185-189.

21. Dichmann DS, Yassin H, Serup P: Analysis of pancreatic endocrine development in GDFII-deficient mice. Dev Dyn 2006 , 235( I I):3016-3025.

22. Esquela AF, Lee SJ: Regulation of metanephric kidney development by growth/differentiation factor II. Dev Biol 2003 , 257(2):356-370.

23. Harmon EB, Apelqvist AA, Smart NG, Gu X, Osborne DH, Kim SK GDF I I modulates NGN3+ islet progenitor cell number and promotes $\beta$-cell differentiation in pancreas development. Development 2004, I 3 I(24):6 I63-6I74.

24. Kim J, Wu HH, Lander AD, Lyons KM, Matzuk MM, Calof AL: GDF I I controls the timing of progenitor cell competence in developing retina. Science 2005, 308(5730): 1927-1930.

25. Wu HH, Ivkovic S, Murray RC, Jaramillo S, Lyons KM, Johnson JE, Calof AL: Autoregulation of neurogenesis by GDFI I. Neuron 2003, 37(2): 197-207.

26. Amthor H, Huang R, McKinnell I, Christ B, Kambadur R, Sharma M Patel $K$ : The regulation and action of myostatin as a negative regulator of muscle development during avian embryogenesis. Dev Biol 2002, 25 I (2):24I-257.

27. Gamer LW, Cox KA, Small C, Rosen V: GdfI I is a negative regulator of chondrogenesis and myogenesis in the developing chick limb. Dev Biol 200I, 229(2):407-420.

28. Zierath JR, Hawley JA: Skeletal muscle fiber type: influence on contractile and metabolic properties. PLoS Biol 2004, 2(1 0):e348.

29. Amthor H, Macharia R, Navarrete R, Schuelke M, Brown SC, Otto A, Voit T, Muntoni F, Vrbova G, Partridge T, et al.: Lack of myostatin results in excessive muscle growth but impaired force generation. Proc Natl Acad Sci USA 2007, 104(6): |835-1840.

30. Girgenrath S, Song K, Whittemore LA: Loss of myostatin expression alters fiber-type distribution and expression of myosin heavy chain isoforms in slow- and fast-type skeletal muscle. Muscle Nerve 2005, 3 I (I):34-40.

31. Souza TA, Chen X, Guo Y, Sava P, Zhang J, Hill JJ, Yaworsky PJ, Qiu $Y$ : Proteomic identification and functional validation of activins and bone morphogenetic protein II as candidate novel muscle mass regulators. Mol Endocrinol 2008 , 22( I 2):2689-2702.

32. Lee SJ: Quadrupling muscle mass in mice by targeting TGF- $\beta$ signaling pathways. PLoS ONE 2007, 2(8):e789.

33. Amthor H, Christ B, Rashid-Doubell F, Kemp CF, Lang E, Patel K: Follistatin regulates bone morphogenetic protein-7 (BMP-7) activity to stimulate embryonic muscle growth. Dev Biol 2002, 243(I): I I5- I 27.

34. de Caestecker $M$ : The transforming growth factor- $\beta$ superfamily of receptors. Cytokine Growth Factor Rev 2004, I 5(I): I-I I.

35. Fainsod A, Deissler K, Yelin R, Marom K, Epstein M, Pillemer G, Steinbeisser $\mathrm{H}$, Blum $M$ : The dorsalizing and neural inducing gene follistatin is an antagonist of BMP-4. Mech Dev 1997, 63(I):39-50.

36. lemura S, Yamamoto TS, Takagi C Uchiyama H, Natsume T, Shimasaki S, Sugino $H$, Ueno N: Direct binding of follistatin to a complex of bone-morphogenetic protein and its receptor inhibits ventral and epidermal cell fates in early Xenopus embryo. Proc Natl Acad Sci USA 1998, 95( I 6):9337-9342.

37. He L, Vichev K, Macharia R, Huang R, Christ B, Patel K, Amthor H: Activin $A$ inhibits formation of skeletal muscle during chick development. Anat Embryol (Berl) 2005, 209(5):40I-407.

38. Oh SP, Li E: The signaling pathway mediated by the type IIB activin receptor controls axial patterning and lateral asymmetry in the mouse. Genes Dev 1997, I I(14): 1812-1826.

39. Rosenthal N, Kornhauser JM, Donoghue M, Rosen KM, Merlie JP: Myosin light chain enhancer activates muscle-specific, developmentally regulated gene expression in transgenic mice. Proc Natl Acad Sci USA 1989, 86(20):7780-7784.

40. Holzenberger M, Lenzner C, Leneuve P, Zaoui R, Hamard G, Vaulont $S$, Bouc YL: Cre-mediated germline mosaicism: a method allowing rapid generation of several alleles of a target gene. Nucleic Acids Res 2000, 28(2I):E92

41. Hayashi S, McMahon AP: Efficient recombination in diverse tissues by a tamoxifen-inducible form of Cre: a tool for temporally regulated gene activation/inactivation in the mouse. Dev Biol 2002, 244(2):305-3 I8
Publish with Bio Med Central and every scientist can read your work free of charge

"BioMed Central will be the most significant development for disseminating the results of biomedical research in our lifetime. "

Sir Paul Nurse, Cancer Research UK

Your research papers will be:

- available free of charge to the entire biomedical community

- peer reviewed and published immediately upon acceptance

- cited in PubMed and archived on PubMed Centra

- yours - you keep the copyright
BioMedcentral 